Acta Crystallographica Section E

Structure Reports

Online

ISSN 1600-5368

\section{Tetrakis $\left(\mu_{3}-2-\{[1,1-b i s(h y d r o x y m e t h y l)-\right.$ 2-oxidoethyl]iminomethyl\}-6-methoxy- phenolato)tetranickel(II) tetrahydrate}

\author{
Yujing Guo, Lianzhi Li,* Yan Liu, Jianfang Dong and Daqi \\ Wang
}

School of Chemistry and Chemical Engineering, Liaocheng University, Shandong 252059, People's Republic of China

Correspondence e-mail: Iilianzhi1963@yahoo.com.cn

Received 4 April 2008; accepted 10 April 2008

Key indicators: single-crystal X-ray study; $T=298 \mathrm{~K}$; mean $\sigma(\mathrm{C}-\mathrm{C})=0.012 \AA$; disorder in main residue; $R$ factor $=0.066 ; w R$ factor $=0.194$; data-to-parameter ratio $=12.9$.

The title complex, $\left[\mathrm{Ni}_{4}\left(\mathrm{C}_{12} \mathrm{H}_{15} \mathrm{NO}_{4}\right)_{4}\right] \cdot 4 \mathrm{H}_{2} \mathrm{O}$, has crystallographic fourfold inversion symmetry, with each $\mathrm{Ni}^{\mathrm{II}}$ ion coordinated in a slightly distorted square-pyramidal coordination environment and forming an $\mathrm{Ni}_{4} \mathrm{O}_{4}$ cubane-like core. In the crystal structure, intermolecular $\mathrm{O}-\mathrm{H} \cdots \mathrm{O}$ hydrogen bonds connect complex and water molecules to form a threedimensional network. The $\mathrm{O}$ atom of one of the unique hydroxymethyl groups is disordered over two sites, with the ratio of occupancies being approximately 0.79:0.21.

\section{Related literature}

For related literature, see: Dong, Li, Xu \& Wang (2007); Dong, Li, Xu, Cui \& Wang (2007); Koikawa et al. (2005); Mishtu et al. (2002); Nihei et al. (2003).

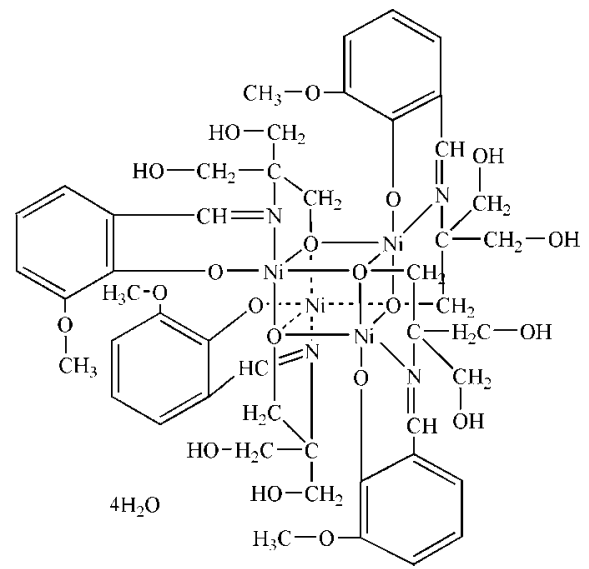

\section{Experimental}

Crystal data

$\left[\mathrm{Ni}_{4}\left(\mathrm{C}_{12} \mathrm{H}_{15} \mathrm{NO}_{4}\right)_{4}\right] \cdot 4 \mathrm{H}_{2} \mathrm{O}$

$M_{r}=1319.90$

Tetragonal, $I 4_{1} / a$

$a=18.754(2) \AA$

$c=15.4395(15) \AA$

$V=5430.3(10) \AA^{3}$

Data collection

Bruker SMART CCD area-detector diffractometer

Absorption correction: multi-scan (SADABS; Sheldrick, 1996)

$T_{\min }=0.670, T_{\max }=0.686$

$Z=4$

Mo $K \alpha$ radiation

$\mu=1.45 \mathrm{~mm}^{-1}$

$T=298(2) \mathrm{K}$

$0.30 \times 0.29 \times 0.28 \mathrm{~mm}$

Refinement

$R\left[F^{2}>2 \sigma\left(F^{2}\right)\right]=0.066$

$w R\left(F^{2}\right)=0.194$

$S=1.08$

2399 reflections

186 parameters

$\mathrm{H}$-atom parameters constrained

$\Delta \rho_{\max }=1.23{\mathrm{e} \AA^{-3}}^{-3}$

$\Delta \rho_{\min }=-0.70 \mathrm{e}^{-3}$

Table 1

Selected geometric parameters $\left(\AA,^{\circ}\right)$.

\begin{tabular}{|c|c|c|c|}
\hline Ni1-O1 & $1.912(4)$ & $\mathrm{Ni} 1-\mathrm{O}^{\mathrm{i}}$ & $1.970(4)$ \\
\hline $\mathrm{Ni} 1-\mathrm{O} 3$ & 1.941 (4) & $\mathrm{Ni} 1-\mathrm{O} 3^{\mathrm{ii}}$ & $2.565(5)$ \\
\hline Ni1-N1 & 1.949 (6) & & \\
\hline $\mathrm{O} 1-\mathrm{Ni} 1-\mathrm{O} 3$ & $172.2(2)$ & $\mathrm{N} 1-\mathrm{Ni} 1-\mathrm{O}^{\mathrm{i}}$ & $166.1(2)$ \\
\hline $\mathrm{O} 1-\mathrm{Ni1}-\mathrm{N} 1$ & $94.3(2)$ & $\mathrm{O} 1-\mathrm{Ni} 1-\mathrm{O}^{\mathrm{ii}}$ & $94.23(17)$ \\
\hline $\mathrm{O} 3-\mathrm{Ni} 1-\mathrm{N} 1$ & $84.1(2)$ & $\mathrm{O} 3-\mathrm{Ni} 1-\mathrm{O}^{\mathrm{ii}}$ & 79.80 (17) \\
\hline $\mathrm{O} 1-\mathrm{Ni} 1-\mathrm{O}^{\mathrm{i}}$ & 94.57 (19) & $\mathrm{N} 1-\mathrm{Ni} 1-\mathrm{O}^{\mathrm{ii}}$ & $117.2(2)$ \\
\hline $\mathrm{O} 3-\mathrm{Ni} 1-\mathrm{O}^{\mathrm{i}}$ & 88.47 (19) & $\mathrm{O} 3^{\mathrm{i}}-\mathrm{Ni} 1-\mathrm{O} 3^{\mathrm{ii}}$ & $72.63(17)$ \\
\hline
\end{tabular}

Symmetry codes: (i) $y-\frac{1}{4},-x+\frac{5}{4},-z+\frac{9}{4}$; (ii) $-x+1,-y+\frac{3}{2}$,

Table 2

Hydrogen-bond geometry $\left(\AA{ }^{\circ}\right)$.

\begin{tabular}{|c|c|c|c|c|}
\hline$D-\mathrm{H} \cdots A$ & $D-\mathrm{H}$ & $\mathrm{H} \cdots A$ & $D \cdots A$ & $D-\mathrm{H} \cdots A$ \\
\hline $\mathrm{O} 5-\mathrm{H} 5 \cdots \mathrm{N} 1$ & 0.82 & 2.58 & $2.988(9)$ & 112 \\
\hline $\mathrm{O} 4-\mathrm{H} 4 \cdots \mathrm{O}^{\mathrm{iii}}$ & 0.82 & 1.94 & $2.714(8)$ & 157 \\
\hline $\mathrm{O} 4^{\prime}-\mathrm{H} 4^{\prime} \cdots \mathrm{O} 6^{\mathrm{iii}}$ & 0.82 & 1.96 & $2.68(3)$ & 148 \\
\hline $\mathrm{O} 6-\mathrm{H} 6 A \cdots \mathrm{O} 1^{\text {iv }}$ & 0.85 & 1.95 & $2.803(7)$ & 180 \\
\hline $\mathrm{O} 6-\mathrm{H} 6 B \cdots \mathrm{O} 4^{\mathrm{v}}$ & 0.85 & 2.04 & $2.892(9)$ & 180 \\
\hline
\end{tabular}

Symmetry codes: (iii) $y-\frac{1}{4},-x+\frac{3}{4}, z+\frac{3}{4}$; (iv) $-y+\frac{5}{4}, x+\frac{1}{4},-z+\frac{5}{4}$; (v) $x, y, z-1$.

Data collection: SMART (Siemens, 1996); cell refinement: SAINT (Siemens, 1996); data reduction: SAINT; program(s) used to solve structure: SHELXS97 (Sheldrick, 2008); program(s) used to refine structure: SHELXL97 (Sheldrick, 2008); molecular graphics: SHELXTL (Sheldrick, 2008); software used to prepare material for publication: SHELXTL.

The authors thank the Natural Science Foundation of Shandong Province (grant No. Y2004B02) for a research grant.

Supplementary data and figures for this paper are available from the IUCr electronic archives (Reference: LH2612). 


\section{metal-organic compounds}

\section{References}

Dong, J.-F., Li, L.-Z., Xu, T., Cui, H. \& Wang, D.-Q. (2007). Acta Cryst. E63, m1501-m1502.

Dong, J.-F., Li, L.-Z., Xu, H.-Y. \& Wang, D.-Q. (2007). Acta Cryst. E63, $\mathrm{m} 2300$.

Koikawa, M., Ohba, M. \& Tokii, T. (2005). Polyhedron, 24, 2257-2262.
Mishtu, D., Chebrolu, P. R., Pauli, K. S. \& Kari, R. (2002). Inorg. Chem. Commun. 5, 380-383.

Nihei, M., Hoshino, N., Ito, T. \& Oshio, H. (2003). Polyhedron, 22, 2359-2362. Sheldrick, G. M. (1996). SADABS. University of Göttingen, Germany.

Sheldrick, G. M. (2008). Acta Cryst. A64, 112-122.

Siemens (1996). SMART and SAINT. Siemens Analytical X-ray Instruments Inc., Madison, Wisconsin, USA. 


\section{supporting information}

Acta Cryst. (2008). E64, m675-m676 [doi:10.1107/S1600536808009872]

\section{Tetrakis $\left(\mu_{3}-2-\{[1,1-b i s(h y d r o x y m e t h y l)-2-o x i d o e t h y l]\right.$ iminomethyl\}-6-methoxy- phenolato)tetranickel(II) tetrahydrate}

\section{Yujing Guo, Lianzhi Li, Yan Liu, Jianfang Dong and Daqi Wang}

\section{S1. Comment}

The chemistry of transition metal ion complexes of hydroxy (aryl-OH and alkyl-OH) rich molecules containing imine/amine group is important in the biomimetic studies of metalloproteins (Mishtu et al., 2002). Polynuclear metal complexes with tridentate ligand containing hydroxyl groups as terminal coordinating atoms have been reported and have attracted much attention (Nihei et al., 2003).

A few structurally characterized multinuclear complexes containing Schiff base ligands has been reported( e.g. Dong, Li, Xu \& Wang (2007); Dong, Li, Xu, Cui \& Wang (2007); Nihei et al., 2003). Herein, we report the synthesis and crystal structure of a novel tetranickel(II) complex with a tridentate Schiff base ligand derived from the condensation of $o$ vanillin and trihydroxymethylaminomethane.

The title compound contains a tetranuclear cubane core based on an approximately cubic array of alternating nickel and oxygen atoms (Fig.1). Each NiI ion is in a distorted square-pyramidal coordination environment with one nitrogen and two oxygen atoms from one Schiff base ligand and two oxygen atoms from the symmetry related units of the cubane core. The Ni atom deviates from the basal plane (formed by O1, N1, O3 and O3, symmetry code (i) $y-7 / 4,-x+3 / 4,-z+$ 7/4) by 0.1299 (33) $\AA$, with a significantly longer $\mathrm{Ni}-\mathrm{O}_{\text {apical }}$ bond distance (Table 1 ). In the molecular structure, the $\mathrm{Ni}-$

Ni distances (3.472 (4) $\AA, 3.182$ (3) $\AA$ ) are longer than some reported values (Koikawa et al., 2005). In addition, there are four $\mathrm{H}_{2} \mathrm{O}$ solvent molecules, which are involved in intermolecular $\mathrm{O}-\mathrm{H} \cdots \mathrm{O}$ hydrogen bonds (Fig. 2, Table 2) which stabilize the crystal atructure along with van der Waals forces.

\section{S2. Experimental}

Trihydroxymethylaminomethane $(1 \mathrm{mmol}, 121.14 \mathrm{mg})$ was dissolved in hot methanol $(10 \mathrm{ml})$ and added successively to a methanol solution $(3 \mathrm{ml})$ of $o$-vanillin $(1 \mathrm{mmol}, 152.15 \mathrm{mg})$. The mixture was then stirred at $323 \mathrm{~K}$ for $2 \mathrm{~h}$. Subsequently, an aqueous solution $(2 \mathrm{ml})$ of nickel chlorate hexahydrate $(1 \mathrm{mmol}, 237.66 \mathrm{mg})$ was added dropwise and stirred for another $5 \mathrm{~h}$. The solution was held at room temperature for ten days, whereupon green blocky crystals suitable for X-ray diffraction were obtained.

\section{S3. Refinement}

Difference Fourier maps revealed that one of the hydroxymethyl group is distorted over two sites. The subsequent refinement of their occupancies gave the value of 0.791 (3) and 0.209 (3), respectively. All the $\mathrm{H}$ atoms were placed in geometrically calculated positions $(\mathrm{C}-\mathrm{H}=0.93-0.97 \AA, \mathrm{O}-\mathrm{H}=0.82 \AA)$ and allowed to ride on their respective parent atoms, with $U_{\text {iso }}(\mathrm{H})=1.2 U_{\text {eq }}(\mathrm{C})$ or $1.5 U_{\text {eq }}\left(\mathrm{C}_{\text {methyl }}\right)$. 


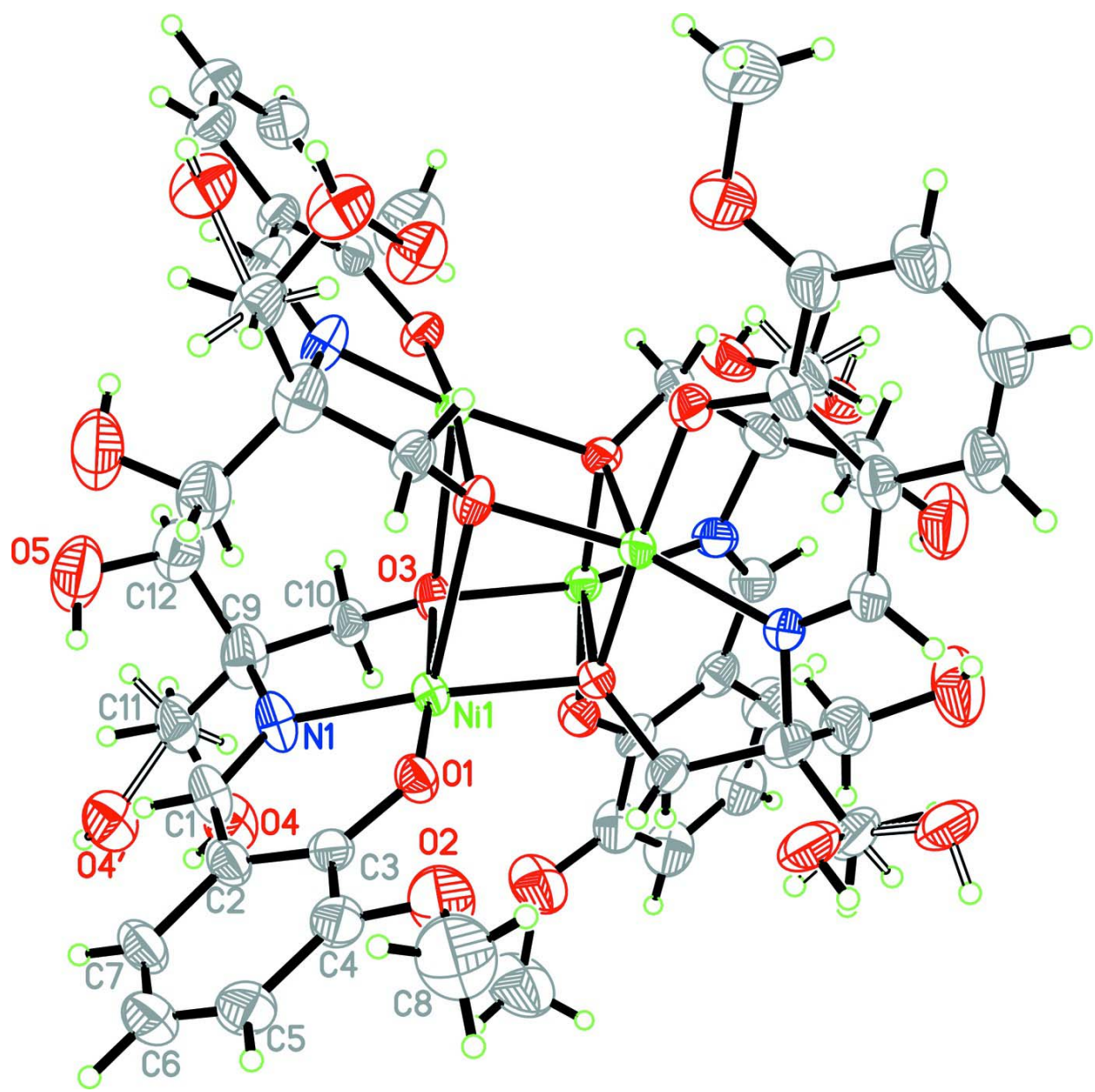

\section{Figure 1}

The structure of the title compound, showing $30 \%$ probability displacement ellipsoids and the atom-numbering scheme. The water solvent molecules are not shown. Open bonds indicate disordered atoms and only the assymetric unit is labelled. 


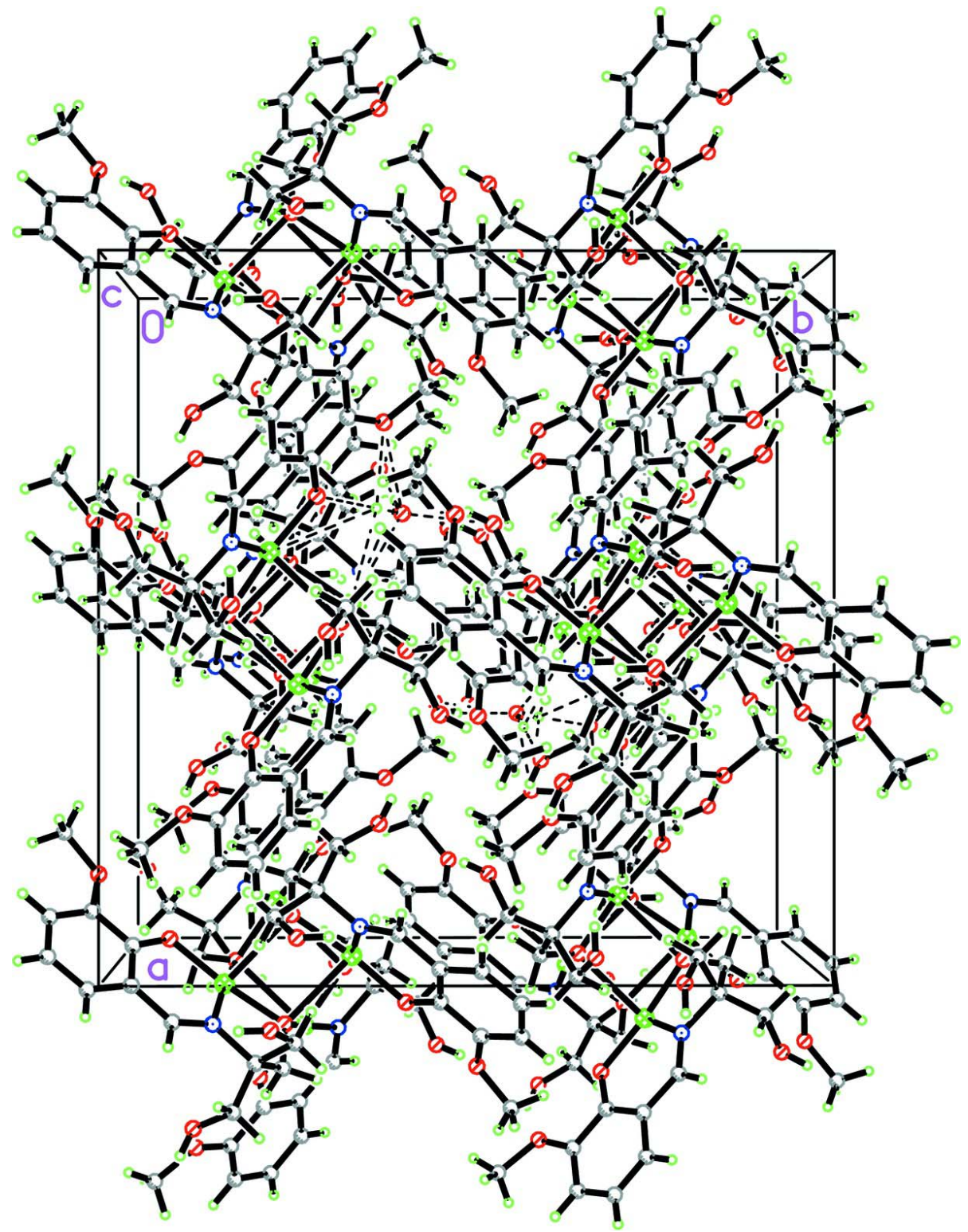

Figure 2

Part of the crystal structure with hydrogen bonds shown as dashed lines. The disorder is not shown.

Tetrakis $\left(\mu_{3}-2-\{[1,1\right.$-bis(hydroxymethyl)-2-oxidoethyl]iminomethyl\}- 6-methoxyphenolato)tetranickel(II) tetrahydrate

Crystal data

$\left[\mathrm{Ni}_{4}\left(\mathrm{C}_{12} \mathrm{H}_{15} \mathrm{NO}_{4}\right)_{4}\right] \cdot 4 \mathrm{H}_{2} \mathrm{O}$

$M_{r}=1319.90$

Tetragonal, $I 4_{1} / a$

Hall symbol: -I $4 \mathrm{ad}$

$a=18.754(2) \AA$

$c=15.4395(15) \AA$

$V=5430.3(10) \AA^{3}$

$D_{\mathrm{x}}=1.614 \mathrm{Mg} \mathrm{m}^{-3}$

$Z=4$

Mo $K \alpha$ radiation, $\lambda=0.71073 \AA$

Cell parameters from 3768 reflections

$\theta=2.2-25.2^{\circ}$

$\mu=1.45 \mathrm{~mm}^{-1}$

$T=298 \mathrm{~K}$

Block, green

$F(000)=2752$

$0.30 \times 0.29 \times 0.28 \mathrm{~mm}$ 


\section{Data collection}

Bruker SMART CCD area-detector diffractometer

Radiation source: fine-focus sealed tube Graphite monochromator

$\varphi$ and $\omega$ scans

Absorption correction: multi-scan (SADABS; Sheldrick, 1996)

$T_{\min }=0.670, T_{\max }=0.686$

\section{Refinement}

Refinement on $F^{2}$

Least-squares matrix: full

$R\left[F^{2}>2 \sigma\left(F^{2}\right)\right]=0.066$

$w R\left(F^{2}\right)=0.194$

$S=1.08$

2399 reflections

186 parameters

0 restraints

Primary atom site location: structure-invariant direct methods
11110 measured reflections

2399 independent reflections

1840 reflections with $I>2 \sigma(I)$

$R_{\text {int }}=0.034$

$\theta_{\max }=25.0^{\circ}, \theta_{\min }=1.7^{\circ}$

$h=-22 \rightarrow 22$

$k=-22 \rightarrow 15$

$l=-9 \rightarrow 18$

Secondary atom site location: difference Fourier map

Hydrogen site location: inferred from neighbouring sites

$\mathrm{H}$-atom parameters constrained

$w=1 /\left[\sigma^{2}\left(F_{\mathrm{o}}{ }^{2}\right)+(0.0801 P)^{2}+60.7787 P\right]$

where $P=\left(F_{\mathrm{o}}^{2}+2 F_{\mathrm{c}}{ }^{2}\right) / 3$

$(\Delta / \sigma)_{\max }=0.001$

$\Delta \rho_{\max }=1.23 \mathrm{e} \AA^{-3}$

$\Delta \rho_{\min }=-0.70$ e $\AA^{-3}$

Special details

Geometry. All e.s.d.'s (except the e.s.d. in the dihedral angle between two 1.s. planes) are estimated using the full covariance matrix. The cell e.s.d.'s are taken into account individually in the estimation of e.s.d.'s in distances, angles and torsion angles; correlations between e.s.d.'s in cell parameters are only used when they are defined by crystal symmetry. An approximate (isotropic) treatment of cell e.s.d.'s is used for estimating e.s.d.'s involving 1.s. planes.

Refinement. Refinement of $F^{2}$ against ALL reflections. The weighted $R$-factor $w R$ and goodness of fit $S$ are based on $F^{2}$, conventional $R$-factors $R$ are based on $F$, with $F$ set to zero for negative $F^{2}$. The threshold expression of $F^{2}>\sigma\left(F^{2}\right)$ is used only for calculating $R$-factors(gt) $e t c$. and is not relevant to the choice of reflections for refinement. $R$-factors based on $F^{2}$ are statistically about twice as large as those based on $F$, and $R$ - factors based on ALL data will be even larger.

Fractional atomic coordinates and isotropic or equivalent isotropic displacement parameters $\left(\AA^{2}\right)$

\begin{tabular}{llllll}
\hline & $x$ & $y$ & $z$ & $U_{\text {iss }} / U_{\text {eq }}$ & Occ. $(<1)$ \\
\hline Ni1 & $0.40964(4)$ & $0.72996(4)$ & $1.05945(5)$ & $0.0374(3)$ & \\
N1 & $0.3971(4)$ & $0.6819(3)$ & $0.9486(4)$ & $0.0575(16)$ & \\
O1 & $0.3327(2)$ & $0.7952(2)$ & $1.0412(3)$ & $0.0480(11)$ & \\
O2 & $0.2316(3)$ & $0.8868(4)$ & $1.0480(5)$ & $0.097(2)$ & \\
O3 & $0.4952(2)$ & $0.6720(2)$ & $1.0675(3)$ & $0.0435(10)$ & \\
O4 & $0.3704(5)$ & $0.5372(4)$ & $0.9346(5)$ & $0.079(2)$ & $0.791(10)$ \\
H4 & 0.3533 & 0.5023 & 0.9103 & $0.119^{*}$ & $0.791(10)$ \\
O4' & $0.3587(16)$ & $0.5547(15)$ & $0.836(2)$ & $0.079(2)$ & $0.209(10)$ \\
H4' & 0.3562 & 0.5134 & 0.8190 & $0.119^{*}$ & $0.209(10)$ \\
O5 & $0.4819(5)$ & $0.6865(5)$ & $0.7848(5)$ & $0.120(3)$ & \\
H5 & 0.4383 & 0.6853 & 0.7893 & $0.181^{*}$ & \\
O6 & $0.3380(3)$ & $0.6042(3)$ & $0.0986(4)$ & $0.0729(16)$ & \\
H6A & 0.3733 & 0.5978 & 0.1322 & $0.088^{*}$ & \\
H6B & 0.3472 & 0.5846 & 0.0503 & $0.088^{*}$ & \\
C1 & $0.3427(4)$ & $0.6889(4)$ & $0.9010(5)$ & $0.061(2)$ & \\
H1 & 0.3392 & 0.6586 & 0.8535 & $0.073^{*}$ &
\end{tabular}




\begin{tabular}{|c|c|c|c|c|c|}
\hline $\mathrm{C} 2$ & $0.2852(4)$ & $0.7394(4)$ & $0.9132(5)$ & 0.0529 (18) & \\
\hline C3 & 0.2839 (3) & $0.7900(4)$ & 0.9815 (4) & $0.0476(16)$ & \\
\hline $\mathrm{C} 4$ & $0.2269(4)$ & $0.8386(5)$ & $0.9823(5)$ & $0.067(2)$ & \\
\hline $\mathrm{C} 5$ & $0.1712(5)$ & $0.8344(6)$ & $0.9218(6)$ & $0.078(3)$ & \\
\hline $\mathrm{H} 5 \mathrm{~A}$ & 0.1334 & 0.8664 & 0.9245 & $0.094^{*}$ & \\
\hline C6 & $0.1727(5)$ & $0.7834(6)$ & $0.8595(6)$ & 0.078 & \\
\hline H6 & 0.1354 & 0.7800 & 0.8201 & $0.094 *$ & \\
\hline $\mathrm{C} 7$ & $0.2279(4)$ & $0.7377(5)$ & $0.8544(6)$ & $0.068(2)$ & \\
\hline H7 & 0.2281 & 0.7038 & 0.8104 & $0.081 *$ & \\
\hline $\mathrm{C} 8$ & $0.1829(7)$ & $0.9466(7)$ & $1.0493(9)$ & $0.130(5)$ & \\
\hline H8A & 0.1797 & 0.9668 & 0.9923 & $0.195^{*}$ & \\
\hline H8B & 0.2001 & 0.9820 & 1.0891 & $0.195^{*}$ & \\
\hline $\mathrm{H} 8 \mathrm{C}$ & 0.1366 & 0.9306 & 1.0675 & $0.195^{*}$ & \\
\hline C9 & $0.4572(5)$ & $0.6290(5)$ & $0.9262(6)$ & $0.075(3)$ & \\
\hline $\mathrm{C} 10$ & $0.4932(4)$ & $0.6144(4)$ & 1.0099 (4) & $0.0523(17)$ & \\
\hline H10A & 0.4691 & 0.5749 & 1.0380 & $0.063^{*}$ & \\
\hline H10B & 0.5418 & 0.5995 & 0.9981 & $0.063^{*}$ & \\
\hline C11 & $0.4273(5)$ & $0.5654(5)$ & $0.8832(6)$ & $0.073(2)$ & \\
\hline H11A & 0.4096 & 0.5781 & 0.8262 & $0.088^{*}$ & $0.791(10)$ \\
\hline H11B & 0.4642 & 0.5296 & 0.8762 & $0.088^{*}$ & $0.791(10)$ \\
\hline $\mathrm{H} 11 \mathrm{C}$ & 0.4635 & 0.5509 & 0.8420 & $0.088^{*}$ & $0.209(10)$ \\
\hline H11D & 0.4276 & 0.5290 & 0.9279 & $0.088^{*}$ & $0.209(10)$ \\
\hline C12 & $0.5136(5)$ & $0.6631(6)$ & $0.8640(6)$ & $0.085(3)$ & \\
\hline $\mathrm{H} 12 \mathrm{~A}$ & 0.5359 & 0.7033 & 0.8927 & $0.101 *$ & \\
\hline H12B & 0.5504 & 0.6283 & 0.8514 & $0.101^{*}$ & \\
\hline
\end{tabular}

Atomic displacement parameters $\left(\AA^{2}\right)$

\begin{tabular}{lllllll}
\hline & $U^{11}$ & $U^{22}$ & $U^{33}$ & $U^{12}$ & $U^{13}$ & $U^{23}$ \\
\hline Ni1 & $0.0427(5)$ & $0.0358(5)$ & $0.0337(5)$ & $0.0002(3)$ & $-0.0072(3)$ & $-0.0007(3)$ \\
N1 & $0.080(4)$ & $0.046(3)$ & $0.046(3)$ & $0.015(3)$ & $-0.022(3)$ & $-0.009(3)$ \\
O1 & $0.045(3)$ & $0.055(3)$ & $0.043(2)$ & $0.008(2)$ & $-0.009(2)$ & $-0.005(2)$ \\
O2 & $0.070(4)$ & $0.125(6)$ & $0.095(5)$ & $0.048(4)$ & $-0.010(4)$ & $-0.019(4)$ \\
O3 & $0.056(3)$ & $0.039(2)$ & $0.036(2)$ & $0.010(2)$ & $-0.008(2)$ & $-0.0013(19)$ \\
O4 & $0.094(6)$ & $0.059(4)$ & $0.086(5)$ & $0.007(4)$ & $-0.005(5)$ & $-0.030(4)$ \\
O4' & $0.094(6)$ & $0.059(4)$ & $0.086(5)$ & $0.007(4)$ & $-0.005(5)$ & $-0.030(4)$ \\
O5 & $0.111(6)$ & $0.189(8)$ & $0.061(4)$ & $0.038(6)$ & $0.005(4)$ & $0.011(5)$ \\
O6 & $0.053(3)$ & $0.096(4)$ & $0.070(4)$ & $-0.001(3)$ & $0.011(3)$ & $-0.003(3)$ \\
C1 & $0.079(5)$ & $0.056(4)$ & $0.048(4)$ & $0.006(4)$ & $-0.022(4)$ & $-0.007(3)$ \\
C2 & $0.055(4)$ & $0.059(4)$ & $0.045(4)$ & $-0.010(3)$ & $-0.013(3)$ & $0.007(3)$ \\
C3 & $0.039(4)$ & $0.059(4)$ & $0.044(4)$ & $-0.001(3)$ & $-0.002(3)$ & $0.014(3)$ \\
C4 & $0.049(4)$ & $0.093(6)$ & $0.058(5)$ & $0.010(4)$ & $-0.001(4)$ & $0.002(5)$ \\
C5 & $0.050(5)$ & $0.110(8)$ & $0.075(6)$ & $0.014(5)$ & $-0.004(4)$ & $0.010(6)$ \\
C6 & $0.060(5)$ & $0.106(7)$ & $0.069(6)$ & $-0.008(5)$ & $-0.018(4)$ & $0.007(5)$ \\
C7 & $0.064(5)$ & $0.081(6)$ & $0.058(5)$ & $-0.011(4)$ & $-0.025(4)$ & $0.005(4)$ \\
C8 & $0.105(9)$ & $0.148(12)$ & $0.137(12)$ & $0.064(9)$ & $-0.013(8)$ & $-0.026(9)$ \\
C9 & $0.088(6)$ & $0.076(6)$ & $0.061(5)$ & $0.035(5)$ & $-0.005(5)$ & $-0.016(4)$ \\
C10 & $0.054(4)$ & $0.060(4)$ & $0.043(4)$ & $0.010(3)$ & $-0.001(3)$ & $-0.013(3)$
\end{tabular}




\begin{tabular}{lllllll}
$\mathrm{C} 11$ & $0.081(6)$ & $0.076(6)$ & $0.061(5)$ & $0.016(5)$ & $-0.007(5)$ & $-0.031(5)$ \\
$\mathrm{C} 12$ & $0.085(7)$ & $0.104(8)$ & $0.065(6)$ & $0.024(6)$ & $0.001(5)$ & $-0.002(5)$ \\
\hline
\end{tabular}

Geometric parameters $\left(A,{ }^{\circ}\right)$

\begin{tabular}{|c|c|c|c|}
\hline $\mathrm{Ni} 1-\mathrm{O} 1$ & $1.912(4)$ & $\mathrm{C} 2-\mathrm{C} 7$ & $1.408(10)$ \\
\hline $\mathrm{Ni} 1-\mathrm{O} 3$ & $1.941(4)$ & $\mathrm{C} 2-\mathrm{C} 3$ & $1.419(10)$ \\
\hline $\mathrm{Ni} 1-\mathrm{N} 1$ & $1.949(6)$ & $\mathrm{C} 3-\mathrm{C} 4$ & $1.405(11)$ \\
\hline $\mathrm{Ni} 1-\mathrm{O}^{\mathrm{i}}$ & $1.970(4)$ & $\mathrm{C} 4-\mathrm{C} 5$ & $1.403(12)$ \\
\hline $\mathrm{Ni} 1-\mathrm{O} 3^{\mathrm{ii}}$ & $2.565(5)$ & $\mathrm{C} 5-\mathrm{C} 6$ & $1.357(13)$ \\
\hline $\mathrm{N} 1-\mathrm{C} 1$ & $1.265(9)$ & $\mathrm{C} 5-\mathrm{H} 5 \mathrm{~A}$ & 0.9300 \\
\hline $\mathrm{N} 1-\mathrm{C} 9$ & $1.541(10)$ & $\mathrm{C} 6-\mathrm{C} 7$ & $1.345(13)$ \\
\hline $\mathrm{O} 1-\mathrm{C} 3$ & $1.303(8)$ & $\mathrm{C} 6-\mathrm{H} 6$ & 0.9300 \\
\hline $\mathrm{O} 2-\mathrm{C} 4$ & $1.362(11)$ & $\mathrm{C} 7-\mathrm{H} 7$ & 0.9300 \\
\hline $\mathrm{O} 2-\mathrm{C} 8$ & $1.446(12)$ & $\mathrm{C} 8-\mathrm{H} 8 \mathrm{~A}$ & 0.9600 \\
\hline $\mathrm{O} 3-\mathrm{C} 10$ & $1.400(8)$ & $\mathrm{C} 8-\mathrm{H} 8 \mathrm{~B}$ & 0.9600 \\
\hline $\mathrm{O} 3-\mathrm{Ni1} 1^{\mathrm{iii}}$ & $1.970(4)$ & $\mathrm{C} 8-\mathrm{H} 8 \mathrm{C}$ & 0.9600 \\
\hline $\mathrm{O} 4-\mathrm{C} 11$ & $1.430(12)$ & $\mathrm{C} 9-\mathrm{C} 11$ & $1.475(13)$ \\
\hline $\mathrm{O} 4-\mathrm{H} 4$ & 0.8200 & $\mathrm{C} 9-\mathrm{C} 10$ & $1.484(11)$ \\
\hline $\mathrm{O} 4-\mathrm{H} 11 \mathrm{D}$ & 1.0883 & $\mathrm{C} 9-\mathrm{C} 12$ & $1.565(14)$ \\
\hline $\mathrm{O} 4^{\prime}-\mathrm{C} 11$ & $1.49(3)$ & $\mathrm{C} 10-\mathrm{H} 10 \mathrm{~A}$ & 0.9700 \\
\hline $\mathrm{O} 4^{\prime}-\mathrm{H} 4^{\prime}$ & 0.8200 & $\mathrm{C} 10-\mathrm{H} 10 \mathrm{~B}$ & 0.9700 \\
\hline $\mathrm{O} 5-\mathrm{C} 12$ & $1.429(12)$ & $\mathrm{C} 11-\mathrm{H} 11 \mathrm{~A}$ & 0.9700 \\
\hline $\mathrm{O} 5-\mathrm{H} 5$ & 0.8200 & $\mathrm{C} 11-\mathrm{H} 11 \mathrm{~B}$ & 0.9700 \\
\hline O6-H6A & 0.8500 & $\mathrm{C} 11-\mathrm{H} 11 \mathrm{C}$ & 0.9698 \\
\hline O6-H6B & 0.8499 & C11-H11D & 0.9699 \\
\hline $\mathrm{C} 1-\mathrm{C} 2$ & $1.447(11)$ & $\mathrm{C} 12-\mathrm{H} 12 \mathrm{~A}$ & 0.9700 \\
\hline $\mathrm{C} 1-\mathrm{H} 1$ & 0.9300 & $\mathrm{C} 12-\mathrm{H} 12 \mathrm{~B}$ & 0.9700 \\
\hline $\mathrm{O} 1-\mathrm{Ni1}-\mathrm{O} 3$ & $172.2(2)$ & $\mathrm{O} 2-\mathrm{C} 8-\mathrm{H} 8 \mathrm{~A}$ & 109.5 \\
\hline $\mathrm{O} 1-\mathrm{Ni1}-\mathrm{N} 1$ & $94.3(2)$ & $\mathrm{O} 2-\mathrm{C} 8-\mathrm{H} 8 \mathrm{~B}$ & 109.5 \\
\hline $\mathrm{O} 3-\mathrm{Ni} 1-\mathrm{N} 1$ & $84.1(2)$ & $\mathrm{H} 8 \mathrm{~A}-\mathrm{C} 8-\mathrm{H} 8 \mathrm{~B}$ & 109.5 \\
\hline $\mathrm{O} 1-\mathrm{Ni} 1-\mathrm{O} 3^{\mathrm{i}}$ & 94.57 (19) & $\mathrm{O} 2-\mathrm{C} 8-\mathrm{H} 8 \mathrm{C}$ & 109.5 \\
\hline $\mathrm{O} 3-\mathrm{Ni} 1-\mathrm{O}^{\mathrm{i}}$ & 88.47 (19) & $\mathrm{H} 8 \mathrm{~A}-\mathrm{C} 8-\mathrm{H} 8 \mathrm{C}$ & 109.5 \\
\hline $\mathrm{N} 1-\mathrm{Ni} 1-\mathrm{O}^{\mathrm{i}}$ & $166.1(2)$ & $\mathrm{H} 8 \mathrm{~B}-\mathrm{C} 8-\mathrm{H} 8 \mathrm{C}$ & 109.5 \\
\hline $\mathrm{O} 1-\mathrm{Ni} 1-\mathrm{O} 3^{\mathrm{ii}}$ & $94.23(17)$ & $\mathrm{C} 11-\mathrm{C} 9-\mathrm{C} 10$ & $114.6(8)$ \\
\hline $\mathrm{O} 3-\mathrm{Ni} 1-\mathrm{O}^{\mathrm{ii}}$ & $79.80(17)$ & $\mathrm{C} 11-\mathrm{C} 9-\mathrm{N} 1$ & $110.2(8)$ \\
\hline $\mathrm{N} 1-\mathrm{Ni} 1-\mathrm{O} 3^{\mathrm{ii}}$ & $117.2(2)$ & $\mathrm{C} 10-\mathrm{C} 9-\mathrm{N} 1$ & $104.9(6)$ \\
\hline $\mathrm{O} 3^{\mathrm{i}}-\mathrm{Ni} 1-\mathrm{O}^{\mathrm{ii}}$ & $72.63(17)$ & $\mathrm{C} 11-\mathrm{C} 9-\mathrm{C} 12$ & $108.1(8)$ \\
\hline $\mathrm{C} 1-\mathrm{N} 1-\mathrm{C} 9$ & $121.7(6)$ & $\mathrm{C} 10-\mathrm{C} 9-\mathrm{C} 12$ & $107.5(8)$ \\
\hline $\mathrm{C} 1-\mathrm{N} 1-\mathrm{Ni} 1$ & $124.1(6)$ & $\mathrm{N} 1-\mathrm{C} 9-\mathrm{C} 12$ & $111.6(7)$ \\
\hline $\mathrm{C} 9-\mathrm{N} 1-\mathrm{Ni} 1$ & $114.0(5)$ & $\mathrm{O} 3-\mathrm{C} 10-\mathrm{C} 9$ & $115.0(6)$ \\
\hline $\mathrm{C} 3-\mathrm{O} 1-\mathrm{Ni} 1$ & $125.9(4)$ & $\mathrm{O} 3-\mathrm{C} 10-\mathrm{H} 10 \mathrm{~A}$ & 108.5 \\
\hline $\mathrm{C} 4-\mathrm{O} 2-\mathrm{C} 8$ & $119.0(8)$ & $\mathrm{C} 9-\mathrm{C} 10-\mathrm{H} 10 \mathrm{~A}$ & 108.5 \\
\hline $\mathrm{C} 10-\mathrm{O} 3-\mathrm{Ni1}$ & $111.7(4)$ & $\mathrm{O} 3-\mathrm{C} 10-\mathrm{H} 10 \mathrm{~B}$ & 108.5 \\
\hline $\mathrm{C} 10-\mathrm{O} 3-\mathrm{Ni} 1^{\mathrm{iii}}$ & $121.9(4)$ & $\mathrm{C} 9-\mathrm{C} 10-\mathrm{H} 10 \mathrm{~B}$ & 108.5 \\
\hline $\mathrm{Ni} 1-\mathrm{O} 3-\mathrm{Ni} 1^{\mathrm{iii}}$ & $108.9(2)$ & $\mathrm{H} 10 \mathrm{~A}-\mathrm{C} 10-\mathrm{H} 10 \mathrm{~B}$ & 107.5 \\
\hline $\mathrm{C} 11-\mathrm{O} 4-\mathrm{H} 4$ & 109.5 & $\mathrm{O} 4-\mathrm{C} 11-\mathrm{C} 9$ & $109.4(7)$ \\
\hline
\end{tabular}




\begin{tabular}{|c|c|c|c|}
\hline $\mathrm{H} 4-\mathrm{O} 4-\mathrm{H} 11 \mathrm{D}$ & 103.2 & $\mathrm{O} 4-\mathrm{C} 11-\mathrm{O} 4^{\prime}$ & $65.0(13)$ \\
\hline $\mathrm{C} 11-\mathrm{O} 4^{\prime}-\mathrm{H}^{\prime}$ & 109.5 & $\mathrm{C} 9-\mathrm{C} 11-\mathrm{O} 4^{\prime}$ & $130.9(13)$ \\
\hline $\mathrm{C} 12-\mathrm{O} 5-\mathrm{H} 5$ & 109.5 & $\mathrm{O} 4-\mathrm{C} 11-\mathrm{H} 11 \mathrm{~A}$ & 109.8 \\
\hline $\mathrm{H} 6 \mathrm{~A}-\mathrm{O} 6-\mathrm{H} 6 \mathrm{~B}$ & 108.4 & C9-C11-H11A & 109.8 \\
\hline $\mathrm{N} 1-\mathrm{C} 1-\mathrm{C} 2$ & $126.4(7)$ & $\mathrm{O} 4{ }^{\prime}-\mathrm{C} 11-\mathrm{H} 11 \mathrm{~A}$ & 45.3 \\
\hline $\mathrm{N} 1-\mathrm{C} 1-\mathrm{H} 1$ & 116.8 & $\mathrm{O} 4-\mathrm{C} 11-\mathrm{H} 11 \mathrm{~B}$ & 109.8 \\
\hline $\mathrm{C} 2-\mathrm{C} 1-\mathrm{H} 1$ & 116.8 & C9-C11-H11B & 109.8 \\
\hline $\mathrm{C} 7-\mathrm{C} 2-\mathrm{C} 3$ & $118.8(7)$ & $\mathrm{O} 4^{\prime}-\mathrm{C} 11-\mathrm{H} 11 \mathrm{~B}$ & 117.9 \\
\hline $\mathrm{C} 7-\mathrm{C} 2-\mathrm{C} 1$ & $118.0(7)$ & $\mathrm{H} 11 \mathrm{~A}-\mathrm{C} 11-\mathrm{H} 11 \mathrm{~B}$ & 108.2 \\
\hline $\mathrm{C} 3-\mathrm{C} 2-\mathrm{C} 1$ & $123.1(6)$ & $\mathrm{O} 4-\mathrm{C} 11-\mathrm{H} 11 \mathrm{C}$ & 141.6 \\
\hline $\mathrm{O} 1-\mathrm{C} 3-\mathrm{C} 4$ & $118.7(7)$ & $\mathrm{C} 9-\mathrm{C} 11-\mathrm{H} 11 \mathrm{C}$ & 104.8 \\
\hline $\mathrm{O} 1-\mathrm{C} 3-\mathrm{C} 2$ & $124.4(6)$ & $\mathrm{O} 4{ }^{\prime}-\mathrm{C} 11-\mathrm{H} 11 \mathrm{C}$ & 104.3 \\
\hline $\mathrm{C} 4-\mathrm{C} 3-\mathrm{C} 2$ & $116.9(7)$ & $\mathrm{H} 11 \mathrm{~A}-\mathrm{C} 11-\mathrm{H} 11 \mathrm{C}$ & 73.3 \\
\hline $\mathrm{O} 2-\mathrm{C} 4-\mathrm{C} 5$ & $125.6(8)$ & $\mathrm{O} 4-\mathrm{C} 11-\mathrm{H} 11 \mathrm{D}$ & 49.5 \\
\hline $\mathrm{O} 2-\mathrm{C} 4-\mathrm{C} 3$ & $112.8(7)$ & C9-C11-H11D & 104.3 \\
\hline $\mathrm{C} 5-\mathrm{C} 4-\mathrm{C} 3$ & $121.6(9)$ & $\mathrm{O} 4^{\prime}-\mathrm{C} 11-\mathrm{H} 11 \mathrm{D}$ & 104.9 \\
\hline $\mathrm{C} 6-\mathrm{C} 5-\mathrm{C} 4$ & $119.7(9)$ & $\mathrm{H} 11 \mathrm{~A}-\mathrm{C} 11-\mathrm{H} 11 \mathrm{D}$ & 145.1 \\
\hline $\mathrm{C} 6-\mathrm{C} 5-\mathrm{H} 5 \mathrm{~A}$ & 120.1 & $\mathrm{H} 11 \mathrm{~B}-\mathrm{C} 11-\mathrm{H} 11 \mathrm{D}$ & 65.7 \\
\hline $\mathrm{C} 4-\mathrm{C} 5-\mathrm{H} 5 \mathrm{~A}$ & 120.1 & $\mathrm{H} 11 \mathrm{C}-\mathrm{C} 11-\mathrm{H} 11 \mathrm{D}$ & 105.4 \\
\hline $\mathrm{C} 7-\mathrm{C} 6-\mathrm{C} 5$ & $120.5(8)$ & $\mathrm{O} 5-\mathrm{C} 12-\mathrm{C} 9$ & $111.7(8)$ \\
\hline $\mathrm{C} 7-\mathrm{C} 6-\mathrm{H} 6$ & 119.8 & $\mathrm{O} 5-\mathrm{C} 12-\mathrm{H} 12 \mathrm{~A}$ & 109.3 \\
\hline $\mathrm{C} 5-\mathrm{C} 6-\mathrm{H} 6$ & 119.8 & $\mathrm{C} 9-\mathrm{C} 12-\mathrm{H} 12 \mathrm{~A}$ & 109.3 \\
\hline $\mathrm{C} 6-\mathrm{C} 7-\mathrm{C} 2$ & $122.3(9)$ & $\mathrm{O} 5-\mathrm{C} 12-\mathrm{H} 12 \mathrm{~B}$ & 109.3 \\
\hline $\mathrm{C} 6-\mathrm{C} 7-\mathrm{H} 7$ & 118.9 & C9- $12-\mathrm{H} 12 \mathrm{~B}$ & 109.3 \\
\hline $\mathrm{C} 2-\mathrm{C} 7-\mathrm{H} 7$ & 118.9 & $\mathrm{H} 12 \mathrm{~A}-\mathrm{C} 12-\mathrm{H} 12 \mathrm{~B}$ & 107.9 \\
\hline $\mathrm{C} 1-\mathrm{C} 2-\mathrm{C} 3-\mathrm{C} 4$ & $176.2(7)$ & & \\
\hline
\end{tabular}

Symmetry codes: (i) $y-1 / 4,-x+5 / 4,-z+9 / 4$; (ii) $-x+1,-y+3 / 2, z$; (iii) $-y+5 / 4, x+1 / 4,-z+9 / 4$.

Hydrogen-bond geometry $\left(A,{ }^{\circ}\right)$

\begin{tabular}{|c|c|c|c|c|}
\hline$D-\mathrm{H} \cdots A$ & $D-\mathrm{H}$ & $\mathrm{H} \cdots A$ & $D \cdots A$ & $D-\mathrm{H} \cdots A$ \\
\hline $\mathrm{O} 5-\mathrm{H} 5 \cdots \mathrm{N} 1$ & 0.82 & 2.58 & $2.988(9)$ & 112 \\
\hline $\mathrm{O} 4-\mathrm{H} 4 \cdots \mathrm{O}^{\mathrm{iv}}$ & 0.82 & 1.94 & $2.714(8)$ & 157 \\
\hline $\mathrm{O} 4^{\prime}-\mathrm{H}^{\prime} \cdots \mathrm{O}^{\mathrm{iv}}$ & 0.82 & 1.96 & $2.68(3)$ & 148 \\
\hline $\mathrm{O} 6-\mathrm{H} 6 A \cdots \mathrm{O} 1^{v}$ & 0.85 & 1.95 & $2.803(7)$ & 180 \\
\hline $\mathrm{O} 6-\mathrm{H} 6 B^{\cdots} \mathrm{O} 4^{\mathrm{vi}}$ & 0.85 & 2.04 & $2.892(9)$ & 180 \\
\hline
\end{tabular}

Symmetry codes: (iv) $y-1 / 4,-x+3 / 4, z+3 / 4$; (v) $-y+5 / 4, x+1 / 4,-z+5 / 4$; (vi) $x, y, z-1$. 